\title{
AMALGAMATION FOR INVERSE AND GENERALIZED INVERSE SEMIGROUPS
}

\author{
T. E. HALL
}

Dedicated to Professor Miyuki Yamada on his 60th birthday

\begin{abstract}
A BSTRACT. For any amalgam $(S, T ; U)$ of inverse semigroups, it is shown that the natural partial order on $S *_{U} T$, the (inverse semigroup) free product of $S$ and $T$ amalgamating $U$, has a simple form on $S \cup T$. In particular, it follows that the semilattice of $S *_{U} T$ is a bundled semilattice of the corresponding semilattice amalgam $(E(S), E(T) ; E(U))$; taken jointly with a result of Teruo Imaoka, this gives that the class of generalized inverse semigroups has the strong amalgamation property. Preserving finiteness is also considered.
\end{abstract}

1. Introduction and summary. A generalized inverse semigroup is a regular semigroup in which the idempotents satisfy $e f g h=e g f h$ (equivalently, the idempotents form a normal band), while a left [right] generalized inverse semigroup is a regular semigroup in which the idempotents satisfy $e f g=e g f[e f g=f e g]$ (equivalently, the idempotents form a left [right] normal band).

By an amalgam of semigroups we mean a list $(S, T ; U)$ of semigroups such that $U$ is a subsemigroup of $S$ and of $T$. We say that $(S, T ; U)$ is strongly embeddable in a semigroup $P$ if there are monomorphisms $\varphi: S \rightarrow P, \psi: T \rightarrow P$ agreeing on $U$ and such that $S \varphi \cap T \psi=U \varphi$.

We say that a class $C$ of semigroups has the strong amalgamation property if every amalgam of semigroups from $C$ is strongly embeddable in a semigroup (depending on the amalgam) from $C$. The definitions of weakly embeddable and the weak amalgamation property are obtained by deleting the condition $S \varphi \cap T \psi=U \varphi$. A special amalgam is one of the form $(S, S ; U)$, that is, one in which $S=T$; and a class $C$ has the special amalgamation property if each special amalgam from $C$ is strongly embeddable in a semigroup from $C$.

Numerous consequences of amalgamation for a class $C$ of algebras (and more generally, relational structures) are given by Jónsson [14]. For inverse semigroups many consequences are given in the author's survey article [4], and a consequence of the embeddability of certain amalgams of regular semigroups is given by the author in [6]. The survey article [15] by Kiss, Márki, Pröhle and Tholen gives a long list of classes of algebras, and topological spaces, with the strong and weak amalgamation properties.

It is known that the class of generalized inverse semigroups has the special amalgamation property (Imaoka [11]) and that any amalgam of generalized inverse semigroups is strongly embeddable in a semigroup (the author [5], and independently, Imaoka): our proof here is independent of these two results.

Received by the editors August 26, 1987.

1980 Mathematics Subject (lassification (1985 Revision). Primary 20M10, 20M17, 20M18. 
In [1] the author showed that the class of inverse semigroups has the strong amalgamation property (see the texts by Howie [10] and Petrich [17] for expositions) and in [11] Imaoka showed that the class of normal bands also has this property. The difficulty in then trying to show that the property holds for the class of generalized inverse semigroups by using Yamada's structure theorem [21] is to match the structure semilattice of a normal band (in which a normal band amalgam is embedded) with the semilattice of an inverse semigroup (in which an inverse semigroup amalgam has been embedded). In the present paper, this difficulty is overcome, by completing the plan of attack due to Imaoka [13].

The key concept is Imaoka's bundled semilattice of an amalgam $\left(S_{1}, S_{2} ; U\right)$ of semilattices, which is a semilattice $S$ in which $\left(S_{1}, S_{2} ; U\right)$ is strongly embedded by monomorphisms $\varphi_{1}: S_{1} \rightarrow S, \varphi_{2}: S_{2} \rightarrow S$ say, such that, for all $s_{1} \in S_{1}$, $s_{2} \in S_{2}$, if $s_{1} \varphi_{1} \leq s_{2} \varphi_{2}$ (in $S$ ) then $s_{1} \leq u$ (in $S_{1}$ ) and $u \leq s_{2}$ (in $S_{2}$ ) for some $u \in U$, and likewise if $s_{2} \varphi_{2} \leq s_{1} \varphi_{1}$ (in $S$ ) then $s_{2} \leq u$ (in $S_{2}$ ) and $u \leq$ $s_{1}$ (in $S_{1}$ ) for some $u \in U$. Imaoka [13] reduced the problem of showing that the class of generalized inverse semigroups has the strong amalgamation property to obtaining an affirmative answer to the following problem: is every amalgam $(S, T ; U)$ of inverse semigroups strongly embeddable in an inverse semigroup $P$ such that $E(P)$, the semilattice of $P$, is thereby a bundled semilattice of the amalgam $(E(S), E(T) ; E(U))$ ?

In $\S 2$ we obtain an affirmative answer to this question. In fact we strongly embed $(S, T ; U)$ in an inverse semigroup $P$ (which is finite if $(S, T ; U)$ is finitely embeddable) such that for all elements $s \in S, t \in T$ (rather than just for idempotents), we have $s \varphi \leq t \psi$ in $P$ if and only if $s \leq u$ in $S$ and $u \leq t$ in $T$ for some $u \in U$, and similarly if $t \psi \leq s \varphi$.

In $\S 3$ we give an example of an amalgam $(S, T ; U)$ of inverse semigroups such that $E(S) *_{E(U)} E(T)$, the free product with amalgamation of the corresponding semilattice amalgam, does not embed in $S{ }^{*} U T$, answering a question of Imaoka [13], posed at the 1979 DeKalb conference on regular semigroups.

In $\S \S 4$ and 5 we give again Imaoka's reduction, with an extra result about a congruence $\Theta$.

In $\S 6$ we consider amalgamation questions for the class of finite generalized inverse semigroups.

2. Embedding amalgams of inverse semigroups. We recall the natural partial order $\leq$ (for example, see [10, Proposition V.2.2]) on an inverse semigroup $S$ defined by $a \leq b$ if $a=a a^{-1} b$.

REMARK 1. There is an alternative proof of the first statement in the next theorem. Its constructions do not preserve finiteness so it does not yield the second statement of the theorem. An outline of this proof is as follows. For any $s \in S$ take the representation $\alpha: S \rightarrow I\left(R_{s}\right)$ given by $x \alpha_{a}=x a$ if $x, x a \in R_{s}$, and $x \alpha_{a}$ is undefined otherwise (this is an orbit of the Preston-Vagner representation). Take the $U$-orbit $X=\left\{s s^{-1} \alpha_{u}: u \in U\right\}$ of the representation $\alpha \mid U$ and extend $(\alpha \mid U)_{X}$, as in the proof of $[\mathbf{1 0}$, Theorem V.8.13], to a representation $\beta: T \rightarrow I(X \cup Y)$. As in [1] or [10, §VII.4], extend the representations $\alpha: S \rightarrow I\left(R_{s}\right)$ and $\beta: T \rightarrow I(X \cup Y)$ simultaneously to a representation of $S *_{U} T$. For $s$ above and any $t \in T$, this representation shows that $s \leq t$ in $S *_{U} T$ if and only if $s \leq u \leq t$ for some $u \in U$. 
THEOREM A. For any amalgam $(S, T ; U)$ of inverse semigroups there is a strong embedding in an inverse semigroup $P$ such that the following conditions are satisfied (without loss of generality, $S$ and $T$ are subsemigroups of $P$ ):

(i) for all $s \in S, t \in T$, if $s \leq t$ in $P$, then $s \leq u$ in $S$ and $u \leq t$ in $T$ for some $u \in U$;

(ii) for all $s \in S, t \in T$, if $t \leq s$ in $P$, then $s \leq u$ in $S$ and $u \leq t$ in $T$ for some $u \in U$.

If the amalgam $(S, T ; U)$ is weakly embeddable in some finite semigroup, then $P$ can be taken to be finite also.

Proof. The strategy of the proof has the following four stages:

(1) reduction to the case of a special amalgam $(V, V ; U)$ of inverse semigroups;

(2) strongly embedding $(V, V ; U)$ in a (noninverse) semigroup $W$, as in the proof of [10, Theorem VII.2.5] (Isbell's Zigzag Theorem), with monomorphisms $\beta: V \rightarrow$ $W, \gamma: V \rightarrow W$ and showing that

(iii) for all $s, t \in V, s \beta=\left[\left(s s^{-1}\right) \beta\right](t \gamma)$ in $W$ implies that $s \leq u \leq t$ in $V$ for some $u \in U$;

(3) altering, as in $[\mathbf{2}, \S 6]$, the strong embedding in stage (2) to a strong embedding in an inverse semigroup $W_{1}$ such that condition (i) of Theorem $\mathrm{A}$ is satisfied;

(4) hence there is also an embedding in an inverse semigroup $W_{2}$ say, satisfying condition (ii), and then the diagonal embedding in $P=W_{1} \times W_{2}$ satisfies both (i) and (ii).

Stage (1). By the weak amalgamation property for inverse semigroups [1], there is an inverse semigroup $V$ and monomorphisms $\varphi: S \rightarrow V, \psi: T \rightarrow V$ which agree on $U$; and if $(S, T ; U)$ is weakly embeddable in a finite semigroup, then by the proof of [2, Theorem 6], $V$ can be taken to be finite. We shall be considering the special amalgam $(V, V ; U \varphi)$, and regarding " $S$ as a subsemigroup of one copy of $V$ and $T$ as a subsemigroup of the other copy of $V$."

Stage (2). The construction here is that of Silver [19] as modified by Stenström [20] to prove Isbell's Zigzag Theorem [10, Theorem VII.2.13]: we use Howie's exposition [10] and his notation (in particular $V^{*}$ and $U^{*}$ denote the semigroups $V$ and $U$ with a common identity 1 adjoined).

Identify $U$ with $U \varphi$ (via $\varphi$ ) and put $A=V^{*} \otimes_{U^{*}} V^{*}$, the tensor product of the $U$-bisystem $V^{*}$ with itself, over $U^{*}$ : then $A$ is also a $U$-bisystem. We consider $\mathbf{Z}_{2}(A)$, the free group of exponent 2 on the set $A$ (rather than $\mathbf{Z}(A)$, to preserve finiteness); we regard $\mathbf{Z}_{2}(A)$ also as a $U^{*}$-bisystem and on the set $W=V \times \mathbf{Z}_{2}(A)$ we define a multiplication [10, Equation VII.2.7] by $(p, x)(q, y)=(p q, p y+x q)$ : here $(p, x)$ can be regarded as denoting the matrix

$$
\left[\begin{array}{ll}
p & x \\
0 & p
\end{array}\right]
$$

The two monomorphisms $\beta$ and $\gamma$ from $V$ into $W$ given by $v \beta=(v, 0)$ and $v \gamma=(v, v \otimes 1-1 \otimes v)$ agree on $U$, and also $V \beta \cap V \gamma=V \beta(=V \gamma)$, by [10, Theorems VII.2.5 and VII.2.14]; we show that this strong embedding of $(V, V ; U)$ in $W$ has property (iii).

So take any $s, t \in V$ such that $s \beta=\left[\left(s s^{-1}\right) \beta\right](t \gamma)$; then

$$
(s, 0)=\left(s s^{-1}, 0\right)(t, t \otimes 1-1 \otimes t),
$$


that is, $(s, 0)=\left(s s^{-1} t, s s^{-1} t \otimes 1-s s^{-1} \otimes t\right)$, so $s=s s^{-1} t$ and $s s^{-1} t \otimes 1=$ $s s^{-1} \otimes t$. This last equation gives us the following zigzag equations, from $\left(s s^{-1} t, 1\right)$ to $\left(s s^{-1}, t\right)$.

There exist elements $x_{1}, x_{2}, \ldots, x_{n+1} \in V^{*}, u_{1}, u_{2}, \ldots, u_{n}, v_{1}, v_{2}, \ldots, v_{n} \in U^{*}$, and $y_{1}, y_{2}, \ldots, y_{n} \in V^{*}$ such that $\left(s s^{-1} t, 1\right)=\left(x_{1}, u_{1} y_{1}\right),\left(s s^{-1}, t\right)=\left(x_{n+1}, v_{n} y_{n}\right)$, and

(1) $x_{i} u_{i}=x_{i+1} v_{i}$, for $i=1,2, \ldots, n$,

(2) $v_{i} y_{i}=u_{i+1} y_{i+1}$, for $i=1,2, \ldots, n-1$.

For $i=1,2, \ldots, n$, we define $w_{i}=u_{1} v_{1}^{-1} u_{2} v_{2}^{-1} \cdots u_{i} v_{i}^{-1}$, an element of $U^{*}$. In the next lemma we deal with a general zigzag from $(x, y)=\left(x_{1}, u_{1} y_{1}\right)$ to $\left(x^{\prime}, y^{\prime}\right)=$ $\left(x_{n+1}, v_{n} y_{n}\right)$.

LEMMA 1. Take any inverse semigroup $U$, any right $U$-system $X$, any left $U$-system $Y$, and any elements $x_{1}, x_{2}, \ldots, x_{n+1} \in X, u_{1}, u_{2}, \ldots, u_{n} \in U$, and $y_{1}, y_{2}, \ldots, y_{n+1} \in Y$, such that $(x, y)=\left(x_{1}, u_{1} y_{1}\right),\left(x^{\prime}, y^{\prime}\right)=\left(x_{n+1}, v_{n} y_{n}\right)$ and equations (1) and (2) hold. Then $w_{n}^{-1} y=w_{n}^{-1} w_{n} y^{\prime}$.

ProOF. From equations (2) alone, we have

$$
\begin{aligned}
w_{n}^{-1} w_{n} y^{\prime} & =w_{n}^{-1} w_{n} v_{n} y_{n} \\
& =v_{n}\left(u_{n}^{-1} w_{n-1}^{-1} w_{n-1} u_{n}\right)\left(v_{n}^{-1} v_{n}\right) y_{n} \\
& =v_{n}\left(v_{n}^{-1} v_{n}\right)\left(u_{n}^{-1} w_{n-1}^{-1} w_{n-1} u_{n}\right) y_{n} \\
& =w_{n}^{-1} w_{n-1} v_{n-1} y_{n-1}=\cdots=w_{n}^{-1} w_{1} v_{1} y_{1} \\
& =v_{n} u_{n}^{-1} \cdots v_{2} u_{2}^{-1} v_{1}\left(u_{1}^{-1} u_{1}\right)\left(v_{1}^{-1} v_{1}\right) y_{1} \\
& =v_{n} u_{n}^{-1} \cdots v_{2} u_{2}^{-1} v_{1}\left(v_{1}^{-1} v_{1}\right)\left(u_{1}^{-1} u_{1}\right) y_{1} \\
& =w_{n}^{-1} u_{1} y_{1}=w_{n}^{-1} y, \quad \text { as required. }
\end{aligned}
$$

REMARK 2. This proof is stage 2 in the proof of [2, Theorem 1] and is generalized in the proof of [5, Lemma $1(\mathrm{i})]$, but is included here for completeness. Using equations (1), we can similarly obtain that $x w_{n}=x^{\prime} w_{n}^{-1} w_{n}$, or this can be deduced from the lemma as a dual result.

We now consider $X=Y=S$, a semigroup containing $U$.

LEMMA 2. If $U$ is an inverse subsemigroup of any semigroup $S$, and if $x_{1}, x_{2}$, $\ldots, x_{n+1}, y_{1}, y_{2}, \ldots, y_{n} \in S$ and $u_{1}, v_{1}, u_{2}, v_{2}, \ldots, u_{n}, v_{n} \in U$, and if equations (1) and $(2)$ hold and $(x, y)=\left(x_{1}, u_{1} y_{1}\right),\left(x^{\prime}, y^{\prime}\right)=\left(x_{n+1}, v_{n} y_{n}\right)$, then $x y=x^{\prime} w_{n}^{-1} w_{n} y^{\prime}$.

PROOF.

$$
\begin{aligned}
x y & =x_{1} u_{1} y_{1}=x_{1} u_{1} u_{1}^{-1} u_{1} y_{1}=x_{2} v_{1} u_{1}^{-1} u_{1} y_{1} \\
& =x_{2} v_{1}\left(v_{1}^{-1} v_{1}\right)\left(u_{1}^{-1} u_{1}\right) y_{1}=x_{2} v_{1}\left(u_{1}^{-1} u_{1}\right)\left(v_{1}^{-1} v_{1}\right) y_{1} \\
& =x_{2} w_{1}^{-1} w_{1} u_{2} y_{2}=x_{2}\left(w_{1}^{-1} w_{1}\right)\left(u_{2} u_{2}^{-1}\right) u_{2} y_{2} \\
& =x_{2}\left(u_{2} u_{2}^{-1}\right)\left(w_{1}^{-1} w_{1}\right) u_{2} y_{2}=x_{3} v_{2}\left(u_{2}^{-1} w_{1}^{-1} w_{1} u_{2}\right) y_{2} \\
& =x_{3} v_{2}\left(v_{2}^{-1} v_{2}\right)\left(u_{2}^{-1} w_{1}^{-1} w_{1} u_{2}\right) y_{2} \\
& =x_{3} v_{2}\left(u_{2}^{-1} w_{1}^{-1} w_{1} u_{2}\right) v_{2}^{-1} v_{2} y_{2} \\
& =x_{3} w_{2}^{-1} w_{2} v_{2} y_{2}=\cdots=x_{n+1}\left(w_{n}^{-1} w_{n}\right) v_{n} y_{n} \\
& =x^{\prime} w_{n}^{-1} w_{n} y^{\prime}, \quad \text { as required. }
\end{aligned}
$$


REMARK 3. This proof is stage 1 in the proof of [2, Theorem 1]. We can obtain similarly that $x^{\prime} y^{\prime}=x w_{n} w_{n}^{-1} y$, or this can be deduced from the lemma by regarding the equations (1) and (2) in reverse order as a zigzag from $\left(x^{\prime}, y^{\prime}\right)$ to $(x, y)$.

We return now to the elements $s, t \in V$, for which $s=s s^{-1} t, s s^{-1} t \otimes 1=s s^{-1} \otimes t$ in $V^{*} \otimes_{U^{*}} V^{*}$, and equations (1) and (2) hold. Lemmas 1 and 2 give us that $w_{n}^{-1}=w_{n}^{-1} w_{n} t$ (that is, $w_{n}^{-1} \leq t$ ) and $s s^{-1} t=s s^{-1} w_{n}^{-1} w_{n} t$ respectively, whence $s=s s^{-1} t=s s^{-1} w_{n}^{-1} w_{n} t=s s^{-1} w_{n}^{-1}$, so $s \leq w_{n}^{-1} \leq t$. Now $w_{n}^{-1} \in U^{*}$ and since $w_{n}^{-1} \leq t \in V=V^{*} \backslash\{1\}$ we have $w_{n}^{-1} \neq 1$, whence $w_{n}^{-1} \in U$. Putting $u=w_{n}^{-1}$ gives us property (iii) as required.

Stage (3). In [2, §6] the author showed how a strong embedding of an amalgam $(S, T ; U)$ of inverse semigroups in a semigroup could be modified to a strong embedding in an inverse semigroup. It can be shown that this modification to our embedding of $(V, V ; U)$ in $W$ gives us an embedding with property (i) of Theorem $\mathrm{A}$, but we shall proceed differently, since a simpler modification of our specific embedding in $W$ will also suffice.

Let $\rho: W \rightarrow I(W)$ denote the right regular representation of $W$ (since $W=W^{1}, \rho$ is faithful) and consider the faithful representations $\beta \rho: V \rightarrow I(W), \gamma \rho: V \rightarrow I(W)$ and $(\beta \rho \mid U): U \rightarrow I(W)$; of course $\beta \rho|U=\gamma \rho| U$. Define the representations $(\beta \rho)^{*}: V \rightarrow I(W),(\gamma \rho)^{*}: V \rightarrow I(W)$ and $(\beta \rho \mid U)^{*}: U \rightarrow I(W)$ as in [18]; for example $(\beta \rho)_{s}^{*}=(\beta \rho)_{s} \mid$ range $(\beta \rho)_{s^{-1}}$ for all $s \in V$. Then $(\beta \rho)^{*},(\gamma \rho)^{*}$ and $(\beta \rho \mid U)^{*}$ are all faithful $[18$, Theorem 1] and clearly all agree on $U$, and so we have a weak embedding of $(V, V ; U)$ in $I(W)$; after proving (i) of Theorem A we shall prove this embedding is a strong embedding.

So we take any $s, t \in V$ such that $(\beta \rho)_{s}^{*} \leq(\gamma \rho)_{t}^{*}$ in $I(W)$, that is, such that $(\beta \rho)_{s}^{*}=(\beta \rho)_{s s^{-1}}^{*}(\gamma \rho)_{t}^{*}$, or equivalently such that

$$
(\beta \rho)_{s} \mid W s^{-1}=\left((\beta \rho)_{s s^{-1}} \mid W s s^{-1}\right)\left((\gamma \rho)_{t} \mid W t\right) .
$$

This gives that $W s^{-1} \subseteq W t$ and $(\gamma \rho)_{t}\left|W s^{-1}=(\beta \rho)_{s}\right| W s^{-1}$ since of course $W s^{-1}=$ $W s s^{-1}$ and $(\beta \rho)_{s s^{-1}} \mid W s s^{-1}$ is the identity map on $W s s^{-1}$. Thus

$$
\begin{aligned}
(\beta \rho)_{s s^{-1}}(\gamma \rho)_{t} & =(\beta \rho)_{s s^{-1}}\left((\gamma \rho)_{t} \mid \operatorname{range}(\beta \rho)_{s s^{-1}}\right) \\
& =(\beta \rho)_{s s^{-1}}\left((\gamma \rho)_{t} \mid W s s^{-1}\right) \\
& =(\beta \rho)_{s s^{-1}}\left((\beta \rho)_{s} \mid W s s^{-1}\right) \\
& =(\beta \rho)_{s s^{-1}}=(\beta \rho)_{s}
\end{aligned}
$$

that is, $\left(\left[\left(s s^{-1}\right) \beta\right](t \gamma)\right) \rho=s \beta \rho$, which gives $s \beta=\left[\left(s s^{-1}\right) \beta\right](t \gamma)$ in $W$ since $\rho$ : $W \rightarrow I\left(W^{1}\right)$ is a monomorphism. Hence, from stage (2), we have that $s \leq u \leq t$ in $V$ for some $u \in U$, giving property (i) for $(V, V ; U)$ with $W_{1}=I(W)$.

Now take any $s, t \in V$ such that $(\beta \rho)_{s}^{*}=(\gamma \rho)_{t}^{*}$ : we show $s=t \in U$. From $(\beta \rho)_{s}^{*} \leq(\gamma \rho)_{t}^{*}$ we get $s \leq u \leq t$ for some $u \in U$, whence $(\beta \rho)_{s}^{*} \leq(\beta \rho)_{u}^{*}=$ $(\gamma \rho)_{u}^{*}=(\gamma \rho)_{t}^{*}=(\beta \rho)_{s}^{*}$ in $I(W)$ and so $(\beta \rho)_{s}^{*}=(\beta \rho)_{u}^{*}$. Since $(\beta \rho)^{*}$ is faithful on $V$, we have $s=u$, and similarly $u=t$, so $s=t \in U$. Thus $(\beta \rho)^{*}: V \rightarrow I(W)$ and $(\gamma \rho)^{*}: V \rightarrow I(W)$ are a strong embedding of $(V, V ; U)$ in $W_{1}=I(W)$ with property (i). Clearly then $\varphi(\beta \rho)^{*}: S \rightarrow I(W)$ and $\psi(\gamma \rho)^{*}: T \rightarrow I(W)$ are a strong embedding of $(S, T ; U)$ in $W_{1}=I(W)$ with property (i).

Stage (4). By the symmetry between $S$ and $T$ in the hypotheses of Theorem A, we deduce the existence of an inverse semigroup $W_{2}$ and monomorphisms $\varphi^{\prime}: S \rightarrow W_{2}$, $\psi^{\prime}: T \rightarrow W_{2}$, agreeing on $U$, such that $S \psi^{\prime} \cap T \psi^{\prime}=U \psi^{\prime}$, and such that property 
(ii) is satisfied. Then the diagonal embeddings $\left(\varphi(\beta \rho)^{*}, \varphi^{\prime}\right): S \rightarrow W_{1} \times W_{2}(s \mapsto$ $\left.\left(s \varphi(\beta \rho)^{*}, s \varphi^{\prime}\right)\right)$ and $\left(\psi(\gamma \rho)^{*}, \psi^{\prime}\right): T \rightarrow W_{1} \times W_{2}\left(t \mapsto\left(t \psi(\beta \rho)^{*}, t \psi^{\prime}\right)\right)$ are a strong embedding of $(S, T ; U)$ in $W_{1} \times W_{2}=P$ satisfying both properties (i) and (ii). If $V$ is finite then $P$ is finite, which completes the proof.

COROLlaRY 1. For any amalgam $(S, T ; U)$ of inverse semigroups, the free product $S *_{U} T$ of $S$ and $T$ amalgamating $U$ has properties (i) and (ii) of Theorem A.

Proof. Take any $s \in S, t \in T$ for which there is no element $u \in U$ such that $s \leq u$ in $S$ and $u \leq t$ in $T$. Then in $P$ we have $s \neq s s^{-1} t$ so in $S *_{U} T$ we also have $s \neq s s^{-1} t$, whence $S *_{U} T$ satisfies property (i), and by symmetry, property (ii) also.

3. A counterexample. We give : example of an amalgam $(S, T ; U)$ of inverse semigroups, with corresponding semilattice amalgam $(E(S), E(T) ; E(U))$, such that $E(S) *_{E(U)} E(T)$ (the free semilattice (or inverse semigroup) product of $E(S)$ and $E(T)$ amalga.nating $E(U))$ does not naturally embed in $S *_{U} T$. This answers negatively Imaoka's Problem 2 of $[\mathbf{1 3}]$ : as noted by Imaoka $[\mathbf{1 3}]$ it is easy to show that $E(S) *_{E(U)} E(T)$ is a bundled semilattice of $(E(S), E(T) ; E(U))$.

Let $\{e, f, g\}$ be the three-element semilattice which is not a chain, with zero element $g$ say, and adjoin an identity 1 to obtain the "diamond" semilattice $E=$ $\{e, f, g, 1\}$. Put $S=T_{E}$, the Munn semigroup of $E[\mathbf{1 0}, \S \mathrm{V} .4]$, and denote by $s$ the nontrivial automorphism of $E$. Then in $S$ we have ses $=s^{-1} e s=f$.

Put $U=\{1, s\}$, the group of units of $S$, and let $T$ be $U$ with an adjoined zero element, $h$ say.

It is easy to see that $E(S) *_{E(U)} E(T)$ is "the cube on a corner," and that $e h, f h, g h$ are all distinct. However, in $S{ }^{*} U T$ these three elements are all equal, since here $f h=s e s h=s e h=s h e=h e=e h$ and $g h=e f h=e e h=e h$.

More generally, for any set $X$ with $|X| \geq 2,\left(I(X), \mathcal{G}(X)^{0} ; \mathcal{G}(X)\right)$ is also a counterexample, where $\mathcal{G}(X)$ is the group of permutations of $X$, and $\mathcal{G}(X)^{0}$ denotes $\mathcal{G}(X)$ with an adjoined zero element.

REMARK 4 . It is easily seen, and well known, that in any variety $\mathcal{V}$ with the weak amalgamation property, $S^{\prime} *_{U} T^{\prime}$ embeds naturally in $S *_{U} T$, where $(S, T ; U)$ is any amalgam from $\mathcal{V}$ and $U \leq S^{\prime} \leq S, U \leq T^{\prime} \leq T$. This is also true in any variety in which $U, S^{\prime}, T^{\prime}$ are weak amalgamation bases.

4. Embedding amalgams of normal bands. In this section we give Imaoka's construction [13] of an embedding of an amalgam of left normal bands in a left normal band $B$ with a prescribed structure semilattice. The new lemma below gives a simpler proof of the validity of the construction.

Pastijn [16] modified Imaoka's construction to deal with strong semilattices (called Plonka sums) of universal algebras, the modification being the replacement of a union of sets by a free product of algebras in a variety: Imaoka's bundled semilattice plays the same crucial role. Though less general, our proof here is simpler since it need not deal with universal algebras.

Take any amalgam $\left(B_{1}, B_{2} ; U\right)$ of left normal bands. Then there is a corresponding amalgam $\left(S_{1}, S_{2} ; V\right)$ of semilattices, and decompositions of $B_{1}, B_{2}, U$ as semilattices of left zero semigroups: $B_{1}=\bigcup_{\alpha \in S_{1}} B_{\alpha}^{1} ; B_{2}=\bigcup_{\alpha \in S_{2}} B_{\alpha}^{2} ;$ and $U=\bigcup_{\alpha \in V} U_{\alpha}$ (where $U_{\alpha}=B_{\alpha}^{1} \cap B_{\alpha}^{2}$, for each $\alpha \in V$ ). For all $\alpha \geq \beta$ in $S_{i}(i=1,2$ ), there is a 
structure mapping $\varphi_{\alpha, \beta}^{i}: B_{\alpha}^{i} \rightarrow B_{\beta}^{i}$ such that the multiplication in $B_{i}$ is given by, for all $\gamma, \delta \in S_{i}$, for all $a \in B_{\gamma}^{i}$ and $b \in B_{\delta}^{i}, a b=a \varphi_{\gamma, \gamma \delta}^{i}$ [10, Proposition IV.5.14].

Take any semilattice $S$ which is a bundled semilattice of the amalgam $\left(S_{1}, S_{2} ; V\right)$. We will prove Imaoka's result that the left normal band amalgam $\left(B_{1}, B_{2} ; U\right)$ is embeddable in a left normal band $B$ whose structure semilattice is $S$. This is achieved in two stages, the first stage dealing with the ideal $I=\{\beta \in S: \beta \leq$ $\alpha$ for some $\left.\alpha \in S_{1} \cup S_{2}\right\}$ of $S$.

Define

$$
E=\left\{(a, \beta) \in\left(S_{1} \cup S_{2}\right) \times I: \alpha \in S_{i}, a \in B_{\alpha}^{i} \text { and } \alpha \geq \beta, i=1,2\right\},
$$

with multiplication $(a, \beta)(b, \gamma)=(a, \beta \gamma)$. Routine checking shows that $E$ is a left normal band with structure semilattice $I$; let its decomposition be $E=\bigcup_{\beta \in I} E_{\beta}$ say. Note that $E_{\beta}$ is in one-to-one correspondence with the nonempty set

$$
\left(\bigcup\left\{B_{\alpha}^{1}: \alpha \geq \beta, \alpha \in S_{1}\right\}\right) \cup\left(\bigcup\left\{B_{\alpha}^{2}: \alpha \geq \beta, \alpha \in S_{2}\right\}\right) .
$$

(It is this union of sets which is replaced by a free product of algebras in Pastijn's modification [16].)

We now define a congruence $\Theta$ on $E$ such that $E / \Theta$ contains the amalgam $\left(B_{1}, B_{2} ; U\right)$ via the mapping $a \mapsto(a, \alpha) \Theta$, where $a \in B_{\alpha}^{i}$.

First we define a relation $\theta$ on $E$ by $(a, \gamma) \theta\left(a \varphi_{\alpha, \beta}^{i}, \gamma\right)$, for $i=1,2$, for $\alpha \geq \beta$ in $S_{i}, \beta \geq \gamma$ in $S$, and for $a \in B_{\alpha}^{i}$. Checking shows that $\theta$ is reflexive and compatible, so $\theta \cup \theta^{-1}$ is likewise, and is also symmetric, so the transitive closure $\left(\theta \cup \theta^{-1}\right)^{t}=\Theta$ say, is a congruence on $E$. (This $\Theta$ is the same as Imaoka's $\theta$ in [12].) Since $\theta \subseteq D(E)$, Green's $D$-relation on $E$, we have that $\Theta \subseteq D(E)$, whence $E / \Theta$ is a normal band with structure semilattice $E / D(E) \simeq I$.

Define, for $i=1,2$, a map $\varphi_{i}: B_{i} \rightarrow E / \Theta$ by $a \varphi_{i}=(a, \alpha) \Theta$, where $\alpha \in S_{i}$ is such that $a \in B_{\alpha}^{i}$. Routine checking shows that $\varphi_{1}, \varphi_{2}$ are morphisms and that $\varphi_{1}\left|U=\varphi_{2}\right| U$. It remains to show that the combined map $\varphi_{1} \cup \varphi_{2}: B_{1} \cup B_{2} \rightarrow E / \Theta$ is one-to-one: the following lemma makes this routine checking. (We note that even though the lemma states that $(a, \alpha) \Theta=(a, \alpha) \theta^{-1} \circ \theta^{-1}$ for certain $(a, \alpha) \in E$, there are simple examples in which $\Theta \neq\left(\theta \cup \theta^{-1}\right)^{n}$, for $\left.n=1,2,3, \ldots\right)$

LEMMA 3. For $i=1,2$, for any $\alpha \in S_{i}$ and any $a \in B_{\alpha}^{i}$, we have $(a, \alpha) \Theta=$ $(a, \alpha) \theta^{-1} \circ \theta^{-1}$.

ProOF. Since $\theta^{-1}$ is reflexive we have $(a, \alpha) \in(a, \alpha) \theta^{-1} \circ \theta^{-1}$ so $(a, \alpha) \Theta \subseteq$ $(a, \alpha) \theta^{-1} \circ \theta^{-1} \circ \Theta \subseteq(a, \alpha) \Theta$ so $(a, \alpha) \theta^{-1} \circ \theta^{-1} \circ \Theta=(a, \alpha) \Theta$. Thus the lemma will follow once we show that (i) $(a, \alpha) \theta^{-1} \circ \theta^{-1} \circ \theta^{-1}=(a, \alpha) \theta^{-1} \circ \theta^{-1}$ and (ii) $(a, \alpha) \theta^{-1} \circ \theta^{-1} \circ \theta=(a, \alpha) \theta^{-1} \circ \theta^{-1}$.

(i) Take any $b, c, d \in B_{1} \cup B_{2}$ such that $(a, \alpha) \theta^{-1}(b, \alpha) \theta^{-1}(c, \alpha) \theta^{-1}(d, \alpha)$. Then we have the situation in Diagram $1(\mathrm{i})$.

If $i=j$ then $c \varphi_{\gamma, \alpha}^{i}=a$ so $(a, \alpha) \theta^{-1}(c, \alpha) \theta^{-1}(d, \alpha)$, and if $j=k$ then $d \varphi_{\delta, \beta}^{j}=b$ so $(a, \alpha) \theta^{-1}(b, \alpha) \theta^{-1}(d, \alpha)$. If $i \neq j \neq k=i$ then $b, c \in B_{i} \cap B_{j}=U$ whence $c \varphi_{\gamma, \beta}^{i}=c \varphi_{\gamma, \beta}^{j}=b$ so $d \varphi_{\delta, \alpha}^{i}=a$ and $(a, \alpha) \theta^{-1}(d, \alpha)$. Thus, in all cases, we have $(a, \alpha) \theta^{-1} \circ \theta^{-1}(d, \alpha)$ as required.

(ii) Take any $b, c, d \in B_{1} \cup B_{2}$ such that $(a, \alpha) \theta^{-1}(b, \alpha) \theta^{-1}(c, \alpha) \theta(d, \alpha)$. Then we have the situation of Diagram 1 (ii), with $\delta \geq \alpha$, and we consider four cases. 


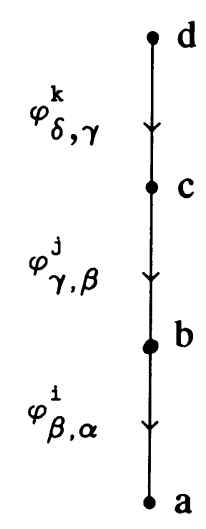

(i) $i=j=k$

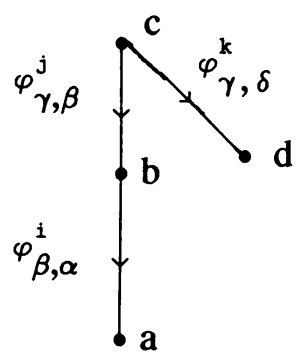

(ii) $i \neq j \neq k$

\section{DIAGRAM 1}

Case 1: $i=j=k$. Then $d \varphi_{\delta, \alpha}^{i}=c \varphi_{\gamma, \alpha}^{i}=a$ whence $(a, \alpha) \theta^{-1}(d, \alpha)$.

Case 2: $i \neq j \neq k(=i)$. Then $b, c \in B_{i} \cap B_{j}=U$ so $c \varphi_{\gamma, \beta}^{i}=c \varphi_{\gamma, \beta}^{j}=b$. Replacing $\varphi_{\gamma, \beta}^{j}$ by $\varphi_{\gamma, \beta}^{i}$ gives us the situation in Case 1 .

Case 3: $i=j \neq k$. Then $c \in B_{j} \cap B_{k}=U$. Since $\alpha \in S_{i}, \delta \in S_{k}$, and $\alpha \leq \delta$, it follows from the bundled semilattice property of $S$ that there exists $\delta^{\prime}$ in $V$ such that $\alpha \leq \delta^{\prime} \leq \delta$. We obtain the situation of Diagram 2(i) since $c \varphi_{\gamma, \delta}^{k} \varphi_{\delta, \delta^{\prime}}^{k}=$ $c \varphi_{\gamma, \delta^{\prime}}^{k}=c \varphi_{\gamma, \delta^{\prime}}^{i}\left(\right.$ from $\left.c \in U, \delta^{\prime} \in V\right)$. Thus $(a, \alpha) \theta^{-1}\left(d \varphi_{\delta, \delta^{\prime}}^{k}, \alpha\right) \theta^{-1}(d, \alpha)$.

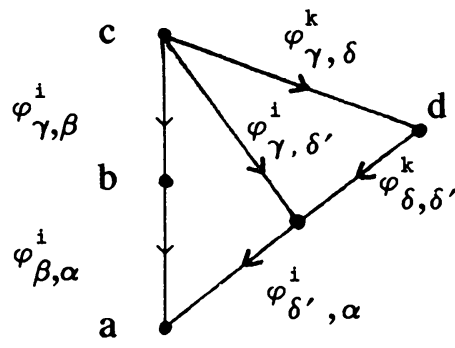

(i) $i=j \neq k$

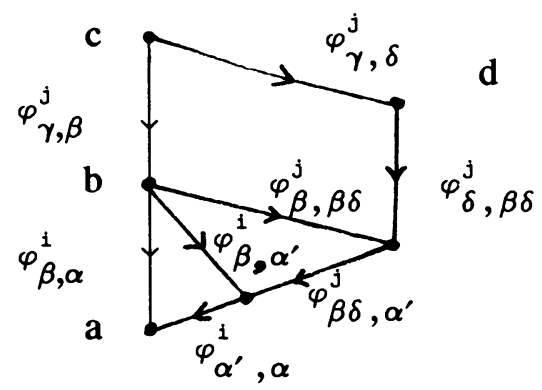

(ii) $i \neq j=k$

\section{DIAGRAM 2}

Case 4: $i \neq j=k$. Then $b \in B_{i} \cap B_{j}=U$. From $\alpha \in S_{i}, \beta \delta \in S_{j}, i \neq j$ and $\alpha \leq \beta \delta$ we have $\alpha \leq \alpha^{\prime} \leq \beta \delta$ for some $\alpha^{\prime} \in V$. We obtain the situation of Diagram 2 (ii) since $b \varphi_{\beta, \beta \delta}^{j} \varphi_{\beta \delta, \alpha^{\prime}}^{j}=b \varphi_{\beta, \alpha^{\prime}}^{j}=b \varphi_{\beta, \alpha^{\prime}}^{i}$. Hence $(a, \alpha) \theta^{-1}\left(d \varphi_{\delta, \alpha^{\prime}}^{j}, \alpha\right) \theta^{-1}(d, \alpha)$.

In all cases we have $(a, \alpha) \theta^{-1} \circ \theta^{-1}(d, \alpha)$, as required, completing the proof of the lemma. 
Routine checking now shows, from the lemma, that $\varphi_{1} \cup \varphi_{2}$ is one-to-one; that is, that each of $\varphi_{1}, \varphi_{2}$ is one-to-one and $B_{1} \varphi_{1} \cap B_{2} \varphi_{2}=U \varphi_{1}$, and so $\left(B_{1}, B_{2} ; U\right)$ is strongly embedded in $E / \Theta$, whose structure semilattice is I.

Now we shall embed $E / \Theta$ into a normal band $B$ with structure semilattice $S$. Let $E / \Theta=\bigcup_{\alpha \in I} F_{\alpha}$ be the semilattice decomposition of $E / \Theta$, with structure maps $\psi_{\alpha, \beta}(\alpha, \beta \in I, \alpha \geq \beta)$ say. For each $\alpha \in S$ now, let $x_{\alpha}$ be an element not in $E / \Theta$ and extend each map $\psi_{\alpha, \beta}(\alpha, \beta \in I, \alpha \geq \beta)$ to a map $\Psi_{\alpha, \beta}: F_{\alpha} \cup\left\{x_{\alpha}\right\} \rightarrow F_{\beta} \cup\left\{x_{\beta}\right\}$ by defining $x_{\alpha} \Psi_{\alpha, \beta}=x_{\beta}$; also, for $\alpha \in S \backslash I, \beta \in S$, with $\alpha \geq \beta$, define $x_{\alpha} \Psi_{\alpha, \beta}=x_{\beta}$. Put $B_{\alpha}=F_{\alpha} \cup\left\{x_{\alpha}\right\}$ for $\alpha \in I$ and $B_{\alpha}=\left\{x_{\alpha}\right\}$ for $\alpha \in S \backslash I$ and put $B=\bigcup_{\alpha \in S} B_{\alpha}$, a left normal band with multiplication given by the structure maps $\Psi_{\alpha, \beta}(\alpha, \beta \in S$, $\alpha \geq \beta$ ). Then $B$ has structure semilattice $S$ and contains $E / \Theta$ and hence also the amalgam $\left(B_{1}, B_{2} ; U\right)$.

THEOREM B (IMAOKA [13, COROLlARY 3]). Take any amalgam $\left(B_{1}, B_{2} ; U\right)$ of [left, right] normal bands. Let $S_{1}, S_{2}, V$, with $S_{1} \cap S_{2}=V$, be the structure semilattices of $B_{1}, B_{2}, U$ respectively, and let $S$ be any bundled semilattice of the amalgam $\left(S_{1}, S_{2} ; U\right)$. Then the amalgam $\left(B_{1}, B_{2} ; U\right)$ is strongly embeddable in $a$ [left, right] normal band $B$ with structure semilattice $S$ and such that $B_{\alpha}^{i} \subseteq B_{\alpha}$ for each $\alpha \in S_{i}$, where $B=\bigcup_{\alpha \in S} B_{\alpha}, B_{1}=\bigcup_{\alpha \in S_{1}} B_{\alpha}^{1}$ and $B_{2}=\bigcup_{\alpha \in S_{2}} B_{\alpha}^{2}$ are the semilattice decompositions.

PROOF. We proved the statement for left normal bands, and the statement for right normal bands is dual. The statement for normal bands follows from these two statements and the decomposition of a normal band $E=\bigcup_{\alpha \in Y} E_{\alpha}$ as the subdirect product $E=\bigcup_{\alpha \in Y} L_{\alpha} \times R_{\alpha}$ of a left normal band $L=\bigcup_{\alpha \in Y} L_{\alpha}$ and a right normal band $R=\bigcup_{\alpha \in Y} R_{\alpha}[10$, Proposition IV.5.22].

REMARK 5 . Imaoka [12] showed by example that the theorem becomes false if the hypothesis that $S$ is a bundled semilattice of $\left(S_{1}, S_{2} ; U\right)$ is replaced by the weaker hypothesis that $\left(S_{1}, S_{2} ; U\right)$ is strongly embedded in $S$.

By putting $B_{1}=B_{2}=U$ we obtain

COROLlARY 2 (IMAOKA [12, LEMMA 1]). Let $S_{1}$ be a subsemilattice of a semilattice $S$ and let $B_{1}=\bigcup_{\alpha \in S_{1}} B_{\alpha}^{1}$ be the semilattice decomposition of $a$ [left, right] normal band $B_{1}$. Then there is a [left, right] normal band $B$ containing $B_{1}$, with structure semilattice $S$, and with semilattice decomposition $B=\bigcup_{\alpha \in S} B_{\alpha}$, such that $B_{\alpha}^{1} \subseteq B_{\alpha}$, for all $\alpha \in S_{1}$.

5. Embedding amalgams of generalized inverse semigroups. Imaoka has shown (see the proof of [12, Theorem 2] for details, and see [13, Corollary 4]) that by combining Theorem A and Imaoka's Theorem B with Yamada's explicit construction [21] of generalized inverse semigroups (in terms of normal bands and inverse semigroups), one easily obtains the following theorem.

THEOREM C (JOINT WITH IMAOKA). The class of [left, right] generalized inverse semigroups has the strong amalgamation property.

6. Preserving finiteness. In this section we ask "when can finiteness be preserved in embedding amalgams of generalized inverse semigroups?"

We gather together relevant results in the literature for inverse and arbitrary semigroups. 
A semigroup $U$ in a class $C$ of semigroups is called a weak [strong] amalgamation base for $C$ if every amalgam of the form $(S, T ; U)$ from $C$ is weakly [strongly] embeddable in a member of $C$.

RESULT 1. (i) [8, THEOREM 10]. The class of finite inverse semigroups has the special amalgamation property.

(ii) (from [2, $\S 6$, last sentence] and (i)). If an amalgam of inverse semigroups is weakly embeddable in a finite semigroup, then it is strongly embeddable in a finite inverse semigroup.

(iii) [3, Remark 1]. If a special amalgam of finite semigroups $(S, S ; U)$ is strongly embeddable in a semigroup, then it is strongly embeddable in a finite semigroup.

(iv) ([7, Theorems 2 and 3 and $\mathbf{9}$, Theorem 3]). A finite inverse semigroup $U$ is a weak [strong] amalgamation base for the class of finite inverse semigroups if and only if $U / J$ is a chain, under the natural ordering of $J$-classes.

Ash's example [1, §3], of an amalgam of finite inverse semigroups not embeddable in any finite semigroup, shows also that the class of finite [left, right] generalized inverse semigroups does not have the weak amalgamation property.

For any amalgam $(S, T ; U)$ of generalized inverse semigroups, let $\left(S^{\prime}, T^{\prime} ; U^{\prime}\right)$ be the corresponding amalgam of inverse semigroups, by which we mean that $S^{\prime} \cap T^{\prime}=$ $U^{\prime}$ and $S^{\prime}, T^{\prime}, U^{\prime}$ are isomorphic to the maximum inverse semigroup images of $S, T, U$ respectively.

THEOREM D. An amalgam $(S, T ; U)$ of finite [left, right] generalized inverse semigroups is strongly [weakly] embeddable in a finite [left, right] generalized inverse semigroup if and only if the corresponding amalgam $\left(S^{\prime}, T^{\prime} ; U^{\prime}\right)$ of inverse semigroups is weakly embeddable in a finite inverse semigroup.

PROOF. (a) "only if" statement. For any orthodox semigroup $P$ and any orthodox subsemigroup $T$, with least inverse semigroup congruences $\mathcal{Y}(P)$ and $\mathcal{Y}(T)$ respectively, it is easily seen from [10, Theorem VI.1.12] that $\mathcal{Y}(T)=\mathcal{Y}(P) \cap(T \times T)$, whence the map $T / \mathcal{Y}(T) \rightarrow P / \mathcal{Y}(P), t \mathcal{Y}(T) \mapsto t \mathcal{Y}(P)$ is well defined and is a monomorphism. The "only if" statement now follows.

(b) "if" statement. Suppose $\left(S^{\prime}, T^{\prime} ; U^{\prime}\right)$ is weakly embeddable in a finite semigroup $V$. By Theorem $\mathrm{A}$, we can embed $\left(S^{\prime}, T^{\prime}, U^{\prime}\right)$ in a finite inverse semigroup $P$ such that $E(P)$ is a bundled semilattice of $\left(E\left(S^{\prime}\right), E\left(T^{\prime}\right) ; E\left(U^{\prime}\right)\right)$. The constructions of $\S \S 4$ and 5 preserve finiteness. Hence $(S, T ; U)$ is strongly embeddable in a finite [left, right] generalized inverse semigroup.

COROLLARY 3. (i) The class of finite [left, right] generalized inverse semigroups has the special amalgamation property.

(ii) Any amalgam of finite [left, right] generalized inverse semigroups which is weakly embeddable in a finite [left, right] generalized inverse semigroup is also strongly embeddable in one.

(iii) A finite [left, right] generalized inverse semigroup $U$ is a weak [strong] amalgamation base for the class of finite [left, right] generalized inverse semigroups if and only if $U / J$ is a chain.

PROOF. To prove (iii) one merely notes that there is a natural isomorphism between the partially ordered sets of $J$-classes of an orthodox semigroup $U$ and its maximum inverse semigroup image $U / \mathcal{Y}(U)$. 
ACKNowledgment. I thank my friend and colleague Chris Ash for a helpful comment and an interesting discussion.

\section{REFERENCES}

1. T. E. Hall, Free products with amalgamation of inverse semigroups, J. Algebra 34 (1975), 375385 .

2. - Representation extension and amalgamation for semigroups, Quart. J. Math. Oxford Ser. (2) 29 (1978), 309-334.

3. _ـ Amalgamation and inverse and regular semigroups, Trans. Amer. Math. Soc. 246 (1978), 395-406.

4. - Inverse and regular semigroups and amalgamation: a brief survey, Proc. Sympos. Regular Semigroups, Northern Illinois Univ., De Kalb, Ill., 1979, pp. 49-79.

5. __ Generalized inverse semigroups and amalgamation, Semigroups (edited by T. E. Hall, P. R. Jones and G. B. Preston), Academic Press, 1980, pp. 145-157.

6. __ On regular semigroups II : an embedding, J. Pure Appl. Algebra 40 (1986), 215-228.

7. __ Finite inverse semigroups and amalgamation, Semigroups and Their Applications (edited by S. M. Goberstein and P. M. Higgins), Reidel, 1987, pp. 51-56.

8. T. E. Hall and P. R. Jones, Epis are onto for finite regular semigroups, Proc. Edinburgh Math. Soc. 26 (1983), 151-162.

9. T. E. Hall and Mohan S. Putcha, The potential J-relation and amalgamation bases for finite semigroups, Proc. Amer. Math. Soc. 95 (1985), 361-364.

10. J. M. Howie, An introduction to semigroup theory, London Math. Soc. Monographs, no. 7, Academic Press, London and New York, 1976.

11. Teruo Imaoka, Free products with amalgamation of bands, Mem. Fac. Lit. Sci. Shimane Univ. Nat. Sci. 10 (1976), 7-17.

12. — The embedding of generalized inverse semigroup amalgams, Mem. Fac. Lit. Sci. Shimane Univ. Nat. Sci. 12 (1978), 1-4.

13. _ - The embedding of regular semigroup amalgams, Proc. Sympos. Regular Semigroups, Northern Illinois Univ., De Kalb, Ill., 1979, pp. 92-100.

14. B. Jónsson, Extensions of relational structures, Theory of Models (Proc. Internat. Sympos. Berkeley, 1963), North-Holland, Amsterdam, 1965, pp. 146-157.

15. E. W. Kiss, L. Márki, P. Pröhle and W. Tholen, Categorical algebraic properties. A compendium on amalgamation, congruence extension, epimorphisms, residual smallness, and injectivity, Studia Sci. Math. Hungar. 18 (1983), 79-141.

16. F. J. Pastijn, Constructions of varieties that satisfy the amalgamation property or the congruence extension property, Studia Sci. Math. Hungar. 17 (1982), 101-111.

17. Mario Petrich, Inverse semigroups, Wiley, 1984.

18. G. B. Preston, Representations of inverse semigroups by one-to-one partial transformations of a set, Semigroup Forum 6 (1973), 240-245, and Addendum, Semigroup Forum 8 (1974), 277.

19. L. Silver, Non-commutative localization and applications, J. Algebra 7 (1967), 44-76.

20. B. Stenström, Flatness and localizations over monoids, Math. Nachr. 48 (1971), 317-333.

21. Miyuki Yamada, Regular semigroups whose idempotents satisfy permutation identities, Pacific $\mathrm{J}$. Math. 21 (1967), 371-392.

Department of Mathematics, Monash University, Clayton, Victoria 3168 , Australia 